\title{
Psychosomatische Grundversorgung in der Praxis - Notwendigkeit und Chance
}

P ro Jahr suchen hierzulande mehr als 40 Millionen Menschen ihren Hausarzt auf. Damit werden in hausärztlichen Praxen weit mehr Patienten betreut als in allen anderen medizinischen Versorgungseinrichtungen. Verschiedene Untersuchungen haben gezeigt, dass bei etwa einem Viertel bis einem Drittel aller Patientenanliegen psychosoziale bzw. psychosomatische Ursachen oder Folgen im Vordergrund stehen. Schon diese Zahlen lassen das (quantitative) Ausmaß des damit aufscheinenden Versorgungsproblems erahnen und weisen auf die Notwendigkeit einer flächendeckenden psychosomatischen Grundversorgung in der Hausarztpraxis hin. Doch damit nicht genug: Die demographische Alterung der Bevölkerung und eine Veränderung des Krankheitsspektrums (der Anteil chronischer Erkrankungen steigt) stellen eine wachsende Herausforderung an die hausärztlich-stützende Begleitung der Betroffenen und ihrer (sie pflegenden) Angehörigen dar. Jeder Hausarzt muss sich den daraus resultierenden Aufgaben einer kontinuierlichen psychosomatischen und somatopsychischen Betreuung täglich neu stellen.

In dieser Ausgabe von psychoneuro soll die psychosomatische Grundversorgung in der hausärztlichen Praxis schlaglichtartig aus eher ungewohnten Blickwinkeln beleuchtet werden. Dabei werden nicht nur psychosomatische Versorgungsprobleme in der Alltagspraxis exemplarisch skizziert (Bahrs mit Hilfe des hermeneutischen Fallverständnisses), sondern vor allem einige chancenreiche und innovative Ansätze zur Optimierung der hausärztlichen Praxis vorgestellt. Am Beispiel der Depression werden sowohl die Potenziale praxisorientierter Diagnose- und Therapieempfehlungen zur vernetzten Versorgung (Härter et al.) als auch eines hausarztpraxisbasierten Case Managements (Gensichen et al.) deutlich. Für eine der schwierigsten (haus)ärztlichen Aufgaben, das Eröffnen einer schwerwiegenden Diagnose, wird ein konkreter, praxisgerechter Weg vorgestellt (Sandholzer).

Insbesondere die Bewältigung chronischer Krankheit könnte das Zukunftsthema der psychosomatischen Versorgung insgesamt werden. Betroffene Patienten und ihre Hausärzte sind dabei auf eine gute, abgestufte Kooperation mit Fachspezialisten aller Versorgungsebenen angewiesen.
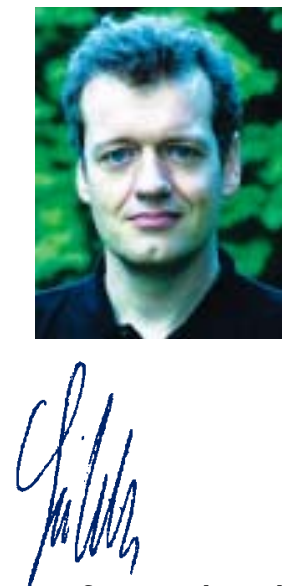

Prof. Dr. med. Ferdinand M. Gerlach, MPH Institut für Allgemeinmedizin der Christian-Albrechts-Universität zu Kiel 